\title{
Squeezing bio-capsules into a constriction: deformation till break-up
}

\author{
A. Le Goff, ${ }^{1}$ B. Kaoui, ${ }^{1, *}$ G. Kurzawa, ${ }^{1}$ B. Haszon, ${ }^{1}$ and A.-V. Salsac ${ }^{1}$ \\ ${ }^{1}$ CNRS, Sorbonne Universités, Université de Technologie de Compiègne, \\ Biomechanics and Bioengineering Laboratory (UMR 7338), 60200 Compiègne, France
}

(Dated: July 17, 2017)

\begin{abstract}
We study experimentally the deformation and break-up of liquid-filled capsules trapped at a step constriction, and subjected to increasing pressure drops. We considered biological (trout fish eggs) and bioartificial (made of ovalbumin and alginate) ones, with the objective to characterize the transition to break-up. We find that both capsule populations are brittle and that the critical pressure drop exhibits a stochastic behavior similar to the rupture of disordered media. The breakup probability follows a Weibull distribution, from which one can deduce the material rupturing characteristics.
\end{abstract}

Problem statement - Capsules consist of an elastic membrane enclosing an inner medium that can be either solid or liquid. They are used to encapsulate, protect and transport an active material and to control its release. For example, microcapsules are used in medicine for targeted drug delivery, to control the local release of a highly strong medicine (e.g. to treat childhood leukemia [1]) or to maintain a constant dose of a drug in chronicle diseases (e.g. insulin for diabetes [2]). Artificial capsules are increasingly used in industry. For example, heat can be managed by a new technology that consists of filling building walls with capsules containing an inner material that absorbs energy during the day and releases heat overnight by undergoing a liquid-to-solid phase transition (Micronal ${ }^{\mathrm{TM}}$ ). Such capsules need to be robust, in order to withstand numerous day-night cycles. Rupture is however desired in other applications, such as in cosmetics. In some formulations, encapsulation is used to protect sensitive active materials from light and oxidation. Break-up is then triggered by forcing the capsuleenriched cream to flow through a narrow pump tube, just prior to application by the consumer (Q10 PLUS Anti-Wrinkle Replenishing Pearls Nivea $\left.{ }^{\mathrm{TM}}\right)$. All these examples demonstrate the challenges in designing capsules with the desired robustness and controlling their rupture, whether it is to be induced or prevented.

Over the last three decades, a large number of studies have investigated the mechanics of capsules theoretically, numerically and experimentally (see the reviews $[3,4]$ and the references therein). The membrane of a capsule experiences three main deformation modes: shearing, compression/dilatation and bending. Each of these deformations costs an energy, which depends on the elastic moduli of the membrane material. Various characterization techniques have been developed to measure the elastic moduli, including parallel plate compression test [5], micropipette aspiration [6], deformation under flow $[7,8]$ to cite a few. Despite its high relevance to industry, the break-up of capsules has received little attention since the pioneering observations of Chang and Olbricht

*Electronic address: badr.kaoui@utc.fr

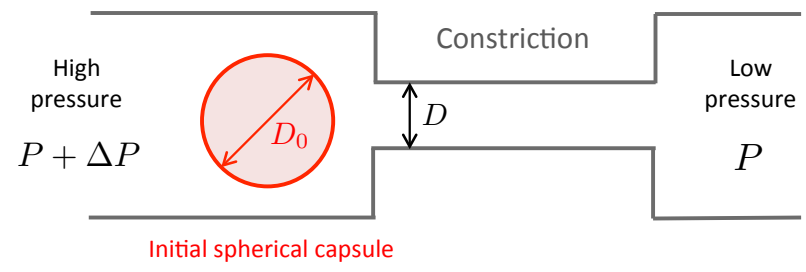

FIG. 1: Geometry of the experimental set-up.

[9], and the more recent study of Koleva and Rehage [10]. Yet the results remain sporadic and do not provide a conclusive understanding of the physics of rupture.

The aim of the present article is to study the breakup of liquid-filled capsules. A constriction is designed to trap capsules, trigger their rupture and characterize their robustness. The channel diameter $D$ after the constriction is chosen to be smaller than the diameter $D_{0}$ of the capsule (see Fig. 1). Depending on the applied pressure drop $\Delta P$ and aspect ratio $D / D_{0}$, the capsule either squeezes into the constriction and flows out of it ('unblocked' case), or gets stuck ('blocked' case), as reported in the state-diagram of Krusters et al. [11]. We show that, by increasing the pressure drop $\Delta P$, starting from the blocked state, we are able to trigger the break-up. The advantages of such a device is to enable a precise control of the release of the encapsulated inner fluid, both in terms of location (at the change in channel confinement) and direction of release of the substance (imposed by the pressure gradient). It also enables a sequential characterization of an entire capsule population. After each test, the channel constriction is washed away by the flow, which cleans the device: it is then ready to characterize the next coming capsule. By analyzing the variability of the critical break-up pressure drop $\Delta P_{C}$ measured in a population, we demonstrate that capsule walls made of a heterogeneous brittle material and we can characterize their robustness.

Experimental setup - Two kinds of capsules are tested. As natural capsules, we use trout fish eggs of the species Oncorhynchus mykiss, sold salted as food products. These eggs have a quasi-spherical shape and exhibit little variation in their size $\left(D_{0}=4.6 \pm 0.2 \mathrm{~mm}\right)$. Know- 

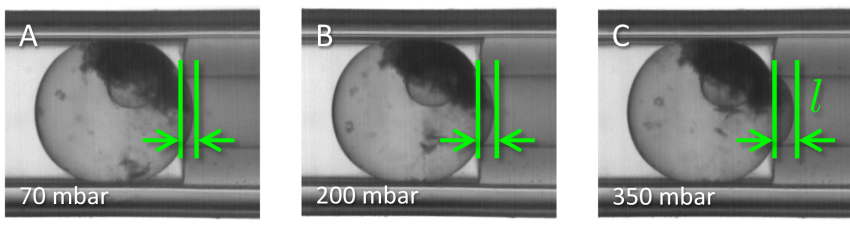

FIG. 2: Deformation of a biological capsule subjected to increasing pressure drops. The penetration length $l$ increases and the capsule is slightly squeezed into the constriction. The inner diameter of the constriction varies from 4.7 to $3.7 \mathrm{~mm}$.

ing that trouts live in both salted and unsalted water, and that the membrane of their eggs is sensitive to variations in salt concentration [12], we manipulate eggs in $\mathrm{NaCl}$ solutions $([\mathrm{NaCl}]=0,6$ or $12 \mathrm{~g} / \mathrm{L})$.

Bioartificial alginate/albumin capsules are prepared by interfacial reticulation following the protocol developed by Edwards-Lévy et al. [13]. Briefly, a solution containing $1 \%$ alginate, $2 \%$ propylene glycol alginate and $8 \%$ ovalbumin is extruded through a nozzle, generating drops that fall into a $10 \%$ calcium chloride bath. After 10 minutes, gelified drops are rinsed 3 times with distilled water and placed in a natrium hydroxide solution $([\mathrm{NaOH}]=1$ to $5 \mathrm{mM}$ ) under agitation for 15 minutes. A membrane is then formed around the beads by transacylation of ovalbumin. The membrane thickness increases with $[\mathrm{NaOH}]$ [5]. The coated beads are rinsed 3 times with distilled water and placed in a $10 \%$ Na-citrate solution for 30 minutes to re-liquify the alginate core. Capsules are then rinsed and manipulated in pure water. This method produces slightly polydisperse populations of spherical capsules.

The experimental setup is depicted in Fig 1. A constriction is built by assembling two transparent cylindrical tubes (made of Tygon or PTFE) and immersing them in a tank filled with water in order to enhance optical quality. A single capsule is placed at the entrance of the constriction, that is itself filled with liquid. Then, a pressure drop is applied between the constriction inlet and outlet using a pressure controller (Elveflow AF1 Premium 1600) connected to the tube entrance. The capsule gets blocked at the constriction entrance.

Then, by increasing the pressure drop step by step the capsule front is forced to squeeze into the constriction, as shown in Fig. 2. After each pressure increment, we either acquire a video of break-up event (at 1500 to 2000 frames per second) or we wait for 30 seconds before recording a snapshot of the deformed capsule, if it is still intact. Images are acquired by the means of a high-speed camera (Photron SA3) equipped with a fixed focal lens (Sigma MACRO $105 \mathrm{~mm}$ ). We measure for each capsule the critical pressure drop $\Delta P_{C}$ at which rupture occurs, and the evolution of the penetration length $l(\Delta P)$ prior to rupture.

Deformation - Prior to rupture $\left(\Delta P<\Delta P_{C}\right)$, the penetration length $l$ quantifies the capsule deformation. Here the control parameter is $\Delta P$, while the measured
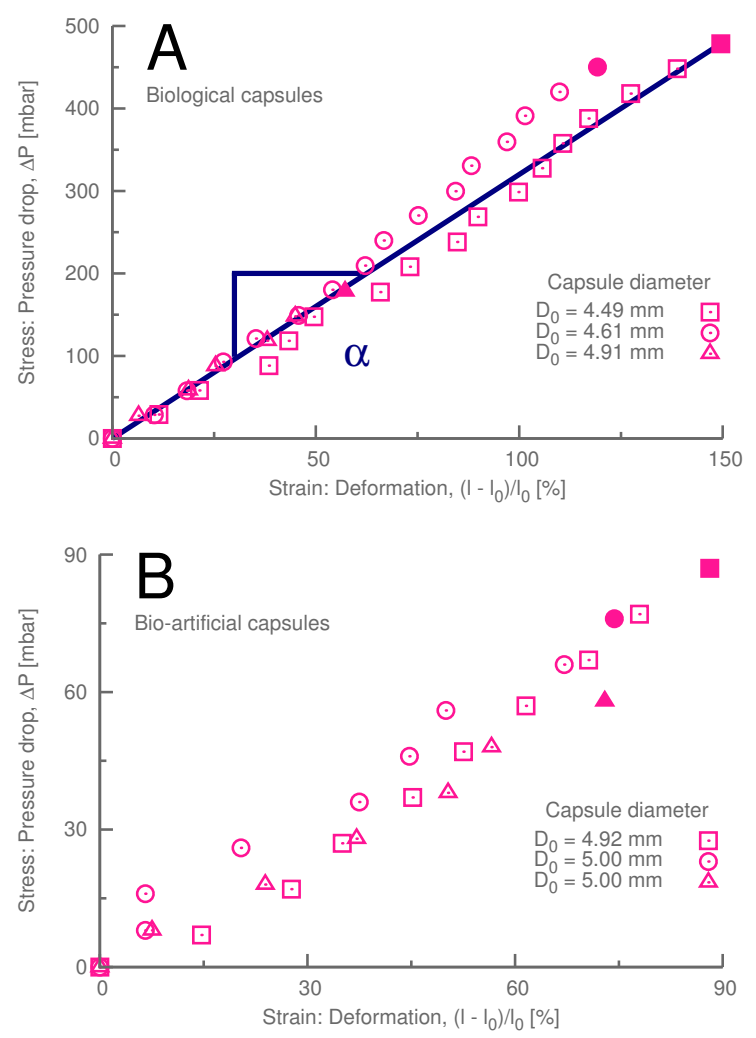

FIG. 3: The applied pressure drop $\Delta P$ versus the deformation of capsules, quantified by $\left(l-l_{0}\right) / l_{0}$ as shown in Fig. 2 , measured for biological capsules (a) and bioartificial capsules (b). The experimental data for different capsules exhibit a linear behavior, which is characteristic of the behavior of an elastic material. The filled symbols represent the last values after which the capsules break up.

observable is $l$. The curve plotted in Fig. 3 can be seen as a stress-strain curve. $\Delta P$ is found to vary linearly with $\left(l-l_{0}\right) / l_{0}$, indicating that the capsules behave as elastic materials (Fig. 3). One must note that higher values of pressure drops are needed to deform the natural capsules than the bioartificial ones. No plastic regime has been observed neither for the biological nor bioartificial capsules. Fitting these experimental data yields a slope $\alpha$, which can be exploited to extract the elastic properties of capsules if used in synergy with a mathematical model describing capsule deformation. $\alpha$ is well conserved for each capsule population. For a given constriction, $\alpha$ is smaller for bioartificial capsules $(\alpha=80-105 \mathrm{mbar})$ than for biological capsules ( $\alpha \simeq 330 \mathrm{mbar}$ ), suggesting that bioartificial capsules are softer. The range of the axes of Fig. 3b is therefore shorter than that of Fig. 3a The last measured data point in each curve, represented by a filled symbol, corresponds to the onset of the capsule break-up $\left(\Delta P_{C}\right)$. We also find that neither $\Delta P_{C}$ nor $\alpha$ are influenced by the salt concentration in the solution. To the best of our knowledge, no theoretical or numerical approach is currently capable of modeling a capsule 

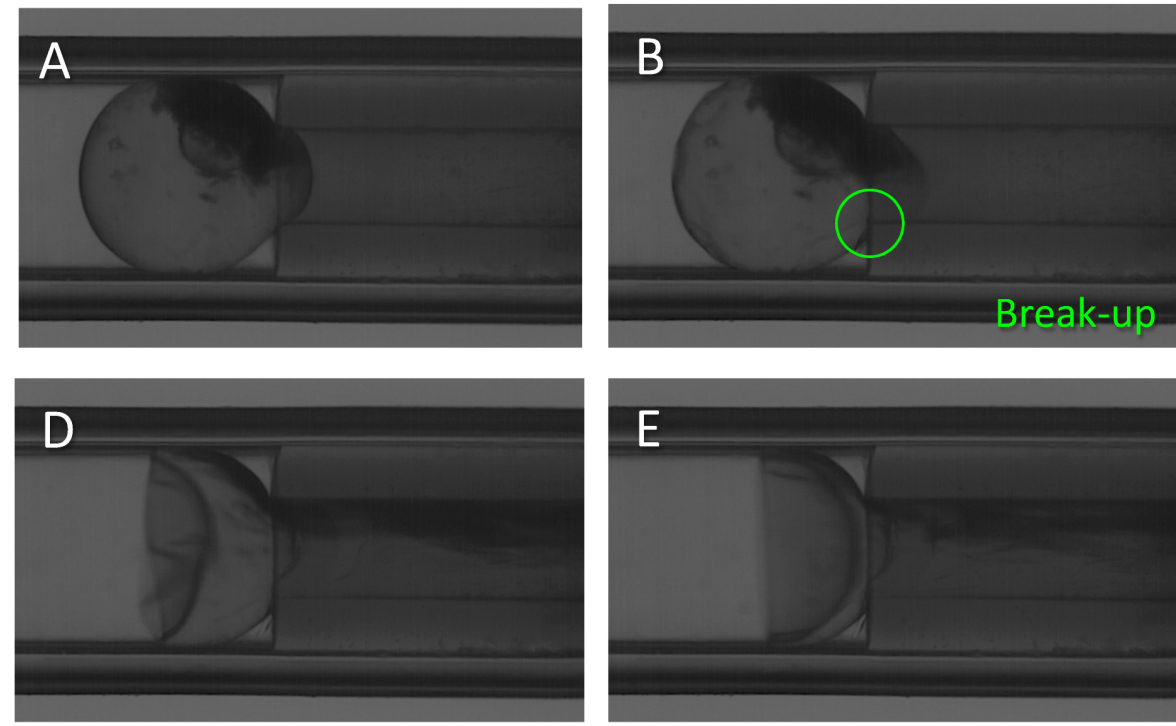
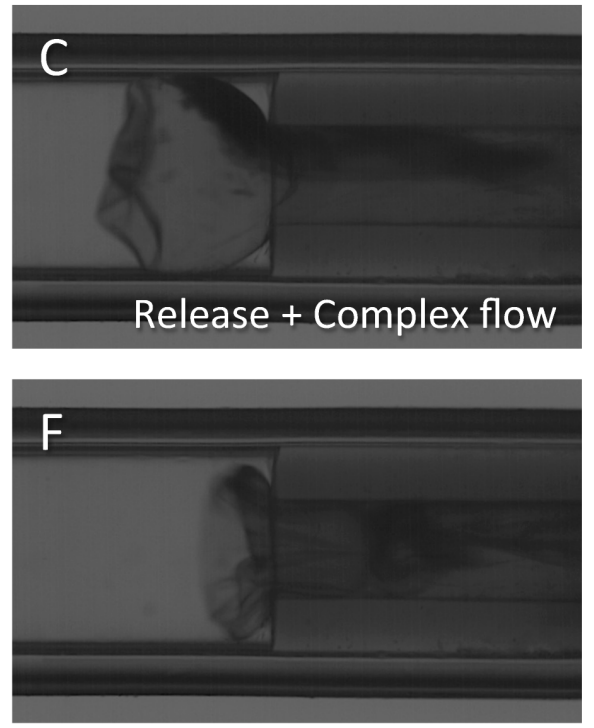

FIG. 4: Snapshots of the break-up scenario of a biological capsule. The fracture (or the crack) of the membrane typically starts from the corner of the constriction as shown by the circle in (b). Upon capsule break-up, the inner content is rapidly released into the downstream post-constriction tube (c-e). Finally, what remains of the capsule is flushed into the constriction (f).

squeezed in a very narrow constriction and dealing with the direct dry contact between the capsule membrane and the constriction walls. Handling properly the contact between a soft particle surface and a rigid wall (in complete absence of a lubricant liquid film) is a challenging numerical task that has not been solved yet.

Break-up and release - Fig. 4 shows a sequence of the break-up event of a capsule. The rupture of the membrane starts typically at the sharp edge of the constriction, where a crack opens. The failure of the capsule reestablishes the overall flow of the ambient fluid between the inlet and the outlet of the constriction. This leads to the instantaneous release of the capsule inner content, within milliseconds, in the form of a transient complex flow into the post-constriction tube. The break-up event occurs in the linear regime of the stress-strain curve, before a plastic regime is reached, which is a signature that the capsule membrane behaves as a brittle material.

What is intriguing here is that using the same experimental set-up and conditions, we do not measure exactly the same critical threshold for the transition to breakup, as shown in Fig. 3 by the filled symbols, although the constant slope in the same graph suggests that capsules in a given population have similar deformability.. In contrast to previous works, such as Ref. [10], that reported a single value for the transition to break-up, we get a cloud of data points of the critical pressure drop for a given capsule size (Fig. 5a). This dispersion in data is observed for both natural and bioartificial capsules.

Such noisy data can be represented in a more meaningful manner by plotting the accumulative probability of the break-up $p$ versus the required critical pressure drop $\Delta P_{C}$ in Fig. $4 \mathrm{~b}$ and fitting with the Weibull distribution
[14]:

$$
p\left(\Delta P_{C}\right)=1-\exp \left[-\left(\frac{\Delta P_{C}-\Delta P_{\mu}}{\Delta P_{0}}\right)^{m}\right],
$$

where $\Delta P_{\mu}, \Delta P_{0}$ and $m$ are the material robustness properties [15]. $\Delta P_{\mu}$ represents the threshold below which all capsules survive, while only $37 \%$ of them are still intact when $\Delta P=\Delta P_{0}+\Delta P_{\mu}$. The Weibull modulus $m$ quantifies the variability of the critical pressure drop. This distribution is popular in the engineering community and classically used to describe the rupture of disordered materials such as glass [16, 17]. In our case, the disordered character is caused by the presence of some defects at the capsule membrane. The membrane is not a perfectly isotropic homogeneous medium. Moreover, we also observe different break-up scenarii. Most capsules break from the corner edge of the inlet of the constriction, while a few rupture at the pole. But, in both cases, we observe the development of a single opening tear: no fragmentation has been recorded, contrary to what is observed for other systems (e.g. for balloons [18]).

Discussion - For capsules used in clinical or industrial contexts, the crucial parameter that is required to be controled is the capsule toughness (called also robustness). In this work, we propose a simple method for characterizing the robustness of fluid-filled soft particles directly, without the need of measuring their mechanical properties, and yielding the minimum value $\Delta P_{\mu}$ below which all capsules remain intact. On the one hand, this proposed technique is a destructive invasive characterization involving the break-up of samples, which is its only drawback. On the other hand, the device can be used to trigger capsule break-up intentionally. Constrictions can be seen as building blocks that could 

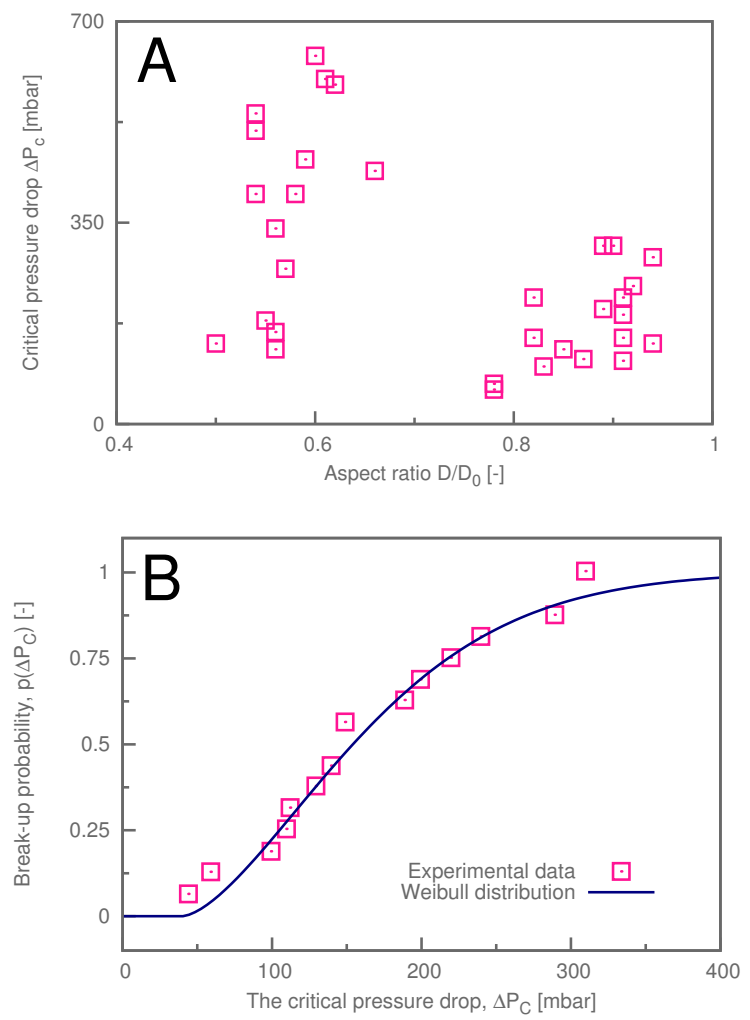

FIG. 5: (a) Critical pressure drop $\Delta P_{C}$ versus the aspect ratio $D / D_{0}$ measured for biological capsules. (b) Accumulative probability $p$ for the capsule to break up at a given critical pressure drop $\Delta P_{C}$. Experimental data (pink square symbols) are fitted with the Weibull distribution formula Eq. (1) (solid blue line) [14]. For the biological capsule population, we get $\Delta P_{\mu}=40$ mbar, $\Delta P_{0}=144$ mbar and $m=1.56$. $\mathrm{s}$ These fitting parameters characterize the toughness (robustness) of the capsule population. be integrated into micro/macro-fluidic reactor devices to trigger capsule break-up and the release of the encapsulated agents in a controlled manner. This may be eventually useful when handling reactive, expensive or toxic substances that need to be manipulated with precaution and at small doses. The present experimental study would greatly benefit from numerical simulations to extract the elastic properties and to compute the pressure and strain distribution in both the fluids and on the membrane. The current challenges are to develop advanced numerical models of capsules that handle the membrane-wall direct dry contact and the break-up by taking into account the change in the capsule surface topology and properly including the physics of rupture [19]. As perspectives, additional intensive experiments need to be carried out to check how the Weibull distribution parameters depend on the constriction geometry and other parameters for possible future standardization of this characterization method.

ALG, BK, GK and AVS acknowledge financial support from Emergence - Sorbonne Universités: 'B3Caps' - Buckling and Breakup of Biocapsules (Grant SU-15R-EMR-11). BH acknowledges support from ERASMUS for her short stay in Compiègne. The authors thank Prof. Dominique Barthès-Biesel and Prof. Daniel Bonamy for fruitful discussions.
[1] V. Krishnan, X. Xu, S. P. Barwe, X. Yang, K. Czymmek, S. A. Waldman, R. W. Mason, X. Jia and A. K. Rajasekaran, Molecular Pharmaceutics 10, 2199 (2013)

[2] K. Chaturvedi, K. Ganguly, M. N. Nadagouda and T. M. Aminabhavi, Journal of Controlled Release 165, 129 (2013)

[3] M. P. Neubauer, M. Poehlmann and A. Fery, Advances in Colloid and Interface Science 207, 65 (2014)

[4] D. Barthès-Biesel, Annu. Rev. Fluid Mech. 48, 25 (2016).

[5] M. Rachik, D. Barthès-Biesel, M. Carin and F. EdwardsLévy, Journal of Colloid and Interface Science 301, 217 (2006)

[6] E. A. Evans, R. Waugh and L. Melnik, Biophysical Journal 16, 585 (1976)

[7] Y. Lefebvre, E. Leclerc, D. Barthès-Biesel, J. Walter and F. Edwards-Lévy, Physics of Fluids 20, 123102 (2008)

[8] P.-Y. Gires, D. Barthès-Biesel, E. Leclerc and A.-V. Salsac, Journal of the Mechanical Behavior of Biomedical Materials 58, 2 (2016)

[9] K. S. Chang and W. L. Olbright, Journal of Fluid Me- chanics 250, 609-633 (1993)

[10] I. Koleva and H. Rehage, Soft Matter 8, 3681(2012)

[11] R. Kusters, T. van der Heijden, B. Kaoui, J. Harting and C. Storm, Physical Review E 90, 033006 (2014)

[12] A. I. Zotin, J. Embryol. Exp. Morph., 6, 546 (1958)

[13] F. Edwards-Lévy and M. C. Lévy, Biomaterials 20, 2069 (1999)

[14] W. Weibull, Journal of Applied Mechanics 18, 293 (1951)

[15] H. J. Herrmann and S. Roux, Statistical Models for the Fracture of disordered Media (North-Holland, Amsterdam, 1990)

[16] X. Brajer, P. Forquin, R. Gy and F. Hild, Journal of Non-Crystalline Solids 316, 42 (2003)

[17] Z. Han, L.C. Tang, J. Xuc and Y. Li, Scripta Materialia 61, 923 (2009)

[18] S. Moulinet and M. Adda-Bedia, Phys. Rev. Lett. 115, 184301 (2015)

[19] D. Bonamy and E. Bouchaud, Physics Reports 498(1), 1 (2011) 